TITLE:

\title{
First-principles XANES simulations of spinel zinc ferrite with a disordered cation distribution
}

\section{$\operatorname{AUTHOR}(\mathrm{S})$ :}

Nakashima, S; Fujita, K; Tanaka, K; Hirao, K; Yamamoto, T; Tanaka, I

\section{CITATION:}

Nakashima, S ... [et al]. First-principles XANES simulations of spinel zinc ferrite with a disordered cation distribution. PHYSICAL REVIEW B 2007, 75(17): 174443.

ISSUE DATE:

2007-05

URL:

http://hdl.handle.net/2433/50197

RIGHT:

Copyright 2007 American Physical Society 
PHYSICAL REVIEW B 75, 174443 (2007)

\title{
First-principles XANES simulations of spinel zinc ferrite with a disordered cation distribution
}

\author{
Seisuke Nakashima, ${ }^{1}$ Koji Fujita, ${ }^{1, *}$ Katsuhisa Tanaka, ${ }^{1}$ Kazuyuki Hirao, ${ }^{1}$ Tomoyuki Yamamoto, ${ }^{2}$ and Isao Tanaka ${ }^{3}$ \\ ${ }^{1}$ Department of Material Chemistry, Graduate School of Engineering, Kyoto University, Nishikyo-ku, Kyoto 615-8510, Japan \\ ${ }^{2}$ Department of Materials Science and Engineering, Waseda University, 3-4-1 Okubo, Shinjuku, Tokyo 169-8555, Japan \\ ${ }^{3}$ Department of Materials Science and Engineering, Graduate School of Engineering, Kyoto University, Sakyo-ku, \\ Kyoto 606-8501, Japan
}

(Received 27 November 2006; published 25 May 2007)

Theoretical calculations of $\mathrm{Zn} K$ and $\mathrm{Fe} K$ x-ray absorption near-edge structures (XANES) using a firstprinciples method have been performed to evaluate the degree of cation disordering in spinel zinc ferrite $\left(\mathrm{ZnFe}_{2} \mathrm{O}_{4}\right)$ thin film prepared by a sputtering method, $\mathrm{ZnFe}_{2} \mathrm{O}_{4}$ thin films annealed at elevated temperatures, and $\mathrm{ZnFe}_{2} \mathrm{O}_{4}$ bulk specimen prepared by a solid-state reaction. Using the full-potential linearized augmented plane-wave + local orbitals method, a theoretical spectrum is generated for the tetrahedral and octahedral environments for each of the two cations. The experimental XANES spectrum of the thin film annealed at $800{ }^{\circ} \mathrm{C}$ as well as that of bulk specimen is successfully reproduced by using either the theoretical spectrum for $\mathrm{Zn}^{2+}$ on the tetrahedral site ( $A$ site) or that for $\mathrm{Fe}^{3+}$ on the octahedral site $(B$ site), which is indicative of the normal spinel structure. For the as-deposited film, on the other hand, excellent agreement between theoretical and experimental spectra is obtained by considering the presence of either ion in both the $A$ and $B$ sites. The degree of cation disordering, $x$, defined as $\left[\mathrm{Zn}_{1-x}{ }^{2+} \mathrm{Fe}_{x}{ }^{3+}\right]_{A}\left[\mathrm{Zn}_{x}{ }^{2+} \mathrm{Fe}_{2-x}{ }^{3+}\right]_{B} \mathrm{O}_{4}$, is estimated to be approximately 0.6 in the as-deposited film, which is consistent with the analysis of the extended x-ray absorption fine structure on the $\mathrm{Zn} K$ edge. Curious magnetic properties as we previously observed for the as-deposited thin film-i.e., ferrimagnetic behaviors accompanied by large magnetization at room temperature and cluster spinglass-like behavior-are discussed in connection with disordering of $\mathrm{Zn}^{2+}$ and $\mathrm{Fe}^{3+}$ ions in the spinel-type structure.

DOI: 10.1103/PhysRevB.75.174443

PACS number(s): 75.70.-i, 78.70.Dm, 81.15.Cd, 82.80.-d

\section{INTRODUCTION}

The stable phase of zinc ferrite $\left(\mathrm{ZnFe}_{2} \mathrm{O}_{4}\right)$ possesses a normal spinel structure-i.e., $\left[\mathrm{Zn}^{2+}\right]_{A}\left[\mathrm{Fe}_{2}{ }^{3+}\right]_{B} \mathrm{O}_{4}$, where the subscripts $A$ and $B$ denote the tetrahedral and octahedral sites in a face-centered-cubic close-packed oxygen sublattice, respectively. It is well known that the normal spinel $\mathrm{ZnFe}_{2} \mathrm{O}_{4}$ behaves like an antiferromagnet with a Néel temperature of $10 \mathrm{~K}$ (strictly speaking, $\mathrm{ZnFe}_{2} \mathrm{O}_{4}$ with ideal normal spinel structure is a three-dimensional spin frustrated magnet, so that the long-range magnetic order is not accomplished down to $1.5 \mathrm{~K}$ at least ${ }^{1,2}$ ). The magnetic transition at very low temperature results from the weak superexchange interaction between $\mathrm{Fe}^{3+}$ ions in the $B$ sites. The fact that the stable phase of $\mathrm{ZnFe}_{2} \mathrm{O}_{4}$ has a normal spinel structure is due to the strong tendency for $\mathrm{Zn}^{2+}$ ions to occupy the $A$ sites in the spinel structure. However, nonequilibrium processing, including mechanical milling, ${ }^{3,4}$ coprecipitation method, ${ }^{5,6}$ rapid quenching, ${ }^{7,8}$ sputtering, ${ }^{9-12}$ and pulsed-laserdeposition (PLD), ${ }^{13}$ induces the metastable phase of $\mathrm{ZnFe}_{2} \mathrm{O}_{4}$ with the arrangement of $\mathrm{Zn}^{2+}$ and $\mathrm{Fe}^{3+}$ ions being disordered. The general formula of disordered $\mathrm{ZnFe}_{2} \mathrm{O}_{4}$ is represented as $\left[\mathrm{Zn}_{1-x}{ }^{2+} \mathrm{Fe}_{x}{ }^{3+}\right]_{A}\left[\mathrm{Zn}_{x}{ }^{2+} \mathrm{Fe}_{2-x}{ }^{3+}\right]_{B} \mathrm{O}_{4}$, where $x$ is an inversion coefficient corresponding to the degree of cation disordering. The magnetic properties of $\mathrm{ZnFe}_{2} \mathrm{O}_{4}$ vary with $x$, depending significantly on the synthetic method and/or postprocessing history. We recently reported that $\mathrm{ZnFe}_{2} \mathrm{O}_{4}$ thin films composed of nanocrystalline particles using the sputtering method exhibit the curious magnetic properties, such as ferrimagnetic properties accompanied by high magnetization even at room temperature, spin freez- ing at a temperature higher than room temperature, and so on. ${ }^{9-12}$ Such behaviors are caused by the formation of the metastable phase via rapid cooling of vapor phases, since the strong superexchange interaction between $\mathrm{Fe}^{3+}$ ions in the $A$ and $B$ sites can lead to the ferrimagnetic properties. Lowtemperature magnetization measurements suggested that $x$ $\sim 2 / 3$; i.e., the chemical formula was very close to $\left[\mathrm{Zn}_{0.33}{ }^{2+} \mathrm{Fe}_{0.67}{ }^{3+}\right]_{A}\left[\mathrm{Zn}_{0.67}{ }^{2+} \mathrm{Fe}_{1.33}{ }^{3+}\right]_{B} \mathrm{O}_{4}$ with completely disordered distribution of $\mathrm{Zn}^{2+}$ and $\mathrm{Fe}^{3+}$ ions, although no distinct proof was provided.

Although many researchers ascribed the ferrimagnetic behaviors observed for the metastable $\mathrm{ZnFe}_{2} \mathrm{O}_{4}$ to cation disordering, only a few quantitative studies have been reported on the degree of cation disordering. By using Rietveld refinement of x-ray diffraction (XRD) data, it is difficult to deduce the degree of cation disordering, due to the similar scattering strength of $\mathrm{Zn}$ and $\mathrm{Fe}$. In the case of nanoparticles such as our samples, the problem is more serious because of the broadness of the diffraction peaks. Mössbauer effect measurements are useful for determining the bond covalence and symmetry around $\mathrm{Fe}$, but provide no direct information on $\mathrm{Zn}$. Because of these, extended x-ray absorption fine structure (EXAFS) analysis, which offers structural parameters concerning ambient atoms, such as coordination numbers and interatomic distance, has been used to determine the degree of cation disordering in various spinel ferrites including NiZn ferrite, ${ }^{14,15} \mathrm{MnZn}$ ferrite,,${ }^{16,17}$ and Cu ferrite. ${ }^{18}$ On the other hand, x-ray absorption near-edge structure (XANES) analysis provides information about the electronic state and chemical bond of a specific atom. Since EXAFS and XANES give us complementary information, the combina- 
tion of both spectral analyses should be more effective in clarifying the local environment of a specific atom. However, the powerful combination was not performed to examine the degree of cation disordering until recently, presumably due to the lack of reliable tools to analyze XANES, in contrast to EXAFS.

Recently, Mo and Ching ${ }^{19,20}$ carried out the firstprinciples orthogonalized linear combinations of atomic orbitals (OLCAO) method for a variety of oxides, such as $\alpha-\mathrm{SiO}_{2}, \alpha-\mathrm{Al}_{2} \mathrm{O}_{3}, \mathrm{MgO}$, and $\mathrm{MgAl}_{2} \mathrm{O}_{4}$, and achieved quantitative reproduction of experimental XANES and energyloss near-edge structure (ELNES) spectra. In their calculation, a core hole is introduced by removing one electron from the core orbital and putting one electron to the unoccupied orbital. Similarly, Tanaka and co-workers ${ }^{21-23}$ demonstrated quantitative reproduction for experimental pre-edge fine structures of $\mathrm{SrTiO}_{3}$ and $\mathrm{BaTiO}_{3}$ as well as for experimental XANES and ELNES spectra of wide-band-gap materials, such as $\mathrm{AlN}, \mathrm{GaN}, \mathrm{InN}, \mathrm{TiO}_{2}$, and $\mathrm{ZnO}$. In addition, they showed that the theoretical calculations of XANES can be used to identify the defect state of ultradilute dopants in ceramics. ${ }^{24}$ Such recent progress in first-principles techniques would make it possible to deduce structural information even in complicated systems such as the metastable $\mathrm{ZnFe}_{2} \mathrm{O}_{4}$.

In this paper, first-principles calculations are carried out in order to obtain the theoretical XANES spectra for $\mathrm{ZnFe}_{2} \mathrm{O}_{4}$ with arbitrary cation disorder and the spectral analysis is utilized to determine the degree of the cation disordering, $x$, in $\mathrm{ZnFe}_{2} \mathrm{O}_{4}$ thin film prepared by the sputtering method, the annealed $\mathrm{ZnFe}_{2} \mathrm{O}_{4}$ thin films, and $\mathrm{ZnFe}_{2} \mathrm{O}_{4}$ bulk specimen prepared by a conventional solid-state reaction. Our particular intention is to make a systematic investigation of the cation disordering and to see if the XANES calculations are really hopeful or hopeless for analyzing the cation disorder from the standpoint of theoretical computations. $\mathrm{ZnFe}_{2} \mathrm{O}_{4}$ is suitable for such purposes because one will be able to alter systematically the cation disorder in the range of $x \sim 0$ (normal spinel) to 2/3 (completely disordered spinel) according to the synthetic procedure, as mentioned above. We demonstrate that the $x$ values derived from first-principles XANES simulations agree well with those from EXAFS analysis. Also, we discuss the magnetic properties of $\mathrm{ZnFe}_{2} \mathrm{O}_{4}$ on the basis of the deduced cation disorder.

\section{METHODOLOGY}

\section{A. Experimental procedure}

A radio-frequency sputtering method was used to prepare $\mathrm{ZnFe}_{2} \mathrm{O}_{4}$ thin films. A film was deposited on a glass substrate (Corning 7059 or silica glass) in an $\mathrm{O}_{2}$ atmosphere (referred to as as-deposited thin film). Some of the resultant thin films were annealed at $350,400,500$, and $800{ }^{\circ} \mathrm{C}$ for $3 \mathrm{~h}$ in air (annealed thin films). The details of the sample preparation were described elsewhere. ${ }^{9,10}$ According to XRD analysis, the as-deposited thin film was composed of $\mathrm{ZnFe}_{2} \mathrm{O}_{4}$ nanocrystals with an average size of $10 \mathrm{~nm}$. It was also ascertained by energy-dispersive $\mathrm{x}$-ray fluorescence spectroscopy that the cation ratio of $\mathrm{Zn}$ to $\mathrm{Fe}$ was 1.07:2, meaning that the

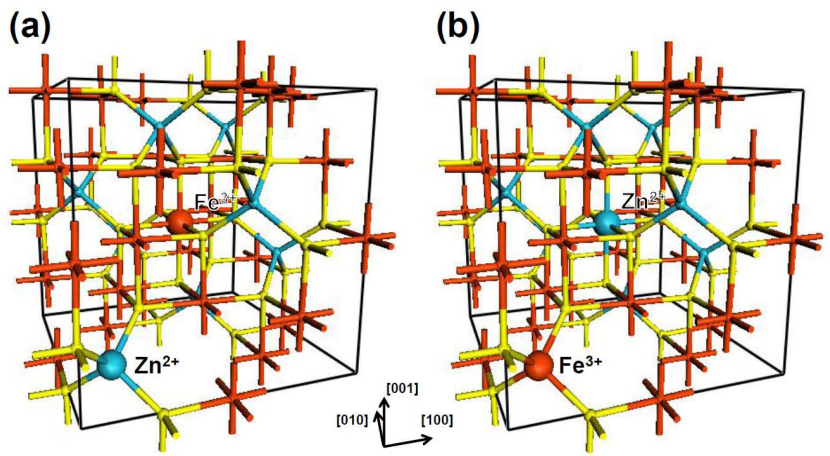

FIG. 1. (Color online) Structural models for XANES simulations: (a) ideal normal spinel structure, and (b) disordered spinel structure containing one tetrahedral $\mathrm{Fe}^{3+}$ ion at $(1 / 81 / 81 / 8)$ and one octahedral $\mathrm{Zn}^{2+}$ ion at $(1 / 21 / 21 / 2)$.

thin film was almost stoichiometric $\mathrm{ZnFe}_{2} \mathrm{O}_{4}$. The thermal annealing at elevated temperatures led to grain growth in a stepwise manner, while keeping a single phase of spinel-type $\mathrm{ZnFe}_{2} \mathrm{O}_{4}$. For comparison, a $\mathrm{ZnFe}_{2} \mathrm{O}_{4}$ polycrystalline pellet prepared by the conventional solid-state reaction was also studied; the mixture prepared from reagent-grade $\mathrm{Fe}_{2} \mathrm{O}_{3}$ and $\mathrm{ZnO}$ was sintered at $1100{ }^{\circ} \mathrm{C}$ for $15 \mathrm{~h}$ in air and then cooled to room temperature at a rate of about $110^{\circ} \mathrm{C} / \mathrm{h}$. The XRD pattern of the resultant pellet revealed a single phase of $\mathrm{ZnFe}_{2} \mathrm{O}_{4}$. The $\mathrm{ZnFe}_{2} \mathrm{O}_{4}$ polycrystal thus obtained will be often called the bulk specimen to distinguish it from thin-film specimens.

Magnetic properties of as-deposited and annealed $\mathrm{ZnFe}_{2} \mathrm{O}_{4}$ thin films and $\mathrm{ZnFe}_{2} \mathrm{O}_{4}$ bulk specimen were investigated using a superconducting quantum interference device (SQUID) magnetometer (Quantum Design, MPMS2). The temperature dependence of dc magnetization was measured under field-cooled (FC) and zero-field-cooled (ZFC) conditions at an external magnetic field of $50 \mathrm{Oe}$.

XANES and EXAFS data at $\mathrm{Zn}-$ and $\mathrm{Fe} K$ absorption edges were obtained for the as-deposited and annealed $\mathrm{ZnFe}_{2} \mathrm{O}_{4}$ thin films and the $\mathrm{ZnFe}_{2} \mathrm{O}_{4}$ bulk specimen utilizing synchrotron radiation from the beamline BL-12 of Photon Factory, National High Energy Institute of Japan. The electron beam energy was $2.5 \mathrm{GeV}$, and the maximum stored current was $330 \mathrm{~mA}$. X-ray absorption spectra were recorded in the X-ray-fluorescence-yield mode at room temperature using a 19-element pure-Ge solid-state detector. Spectral data were collected in the range of $9200.0-10760.0 \mathrm{eV}$ for $\mathrm{Zn}$ and in the range of $6900.0-7260.0 \mathrm{eV}$ for $\mathrm{Fe}$ using an $\mathrm{Si}(111)$ double-crystal monochromator.

\section{B. Computational procedure}

First-principles calculations based on density functional theory have been carried out to simulate XANES spectra using the full-potential linearized augmented plane wave + local orbitals $($ FLAPW + lo) package WIEN2k. Two kinds of spinel-type structure were adopted for the theoretical calculation as illustrated in Fig. 1. One is the ideal normal spinel $\mathrm{ZnFe}_{2} \mathrm{O}_{4}$ structure with space group $F d 3 m$ [Fig. 1(a)] and the other the $\mathrm{ZnFe}_{2} \mathrm{O}_{4}$ with cation disorder [Fig. 1(b)], with a 
$\mathrm{Zn}^{2+}$ ion residing on the $B$ site at $(1 / 21 / 21 / 2)$, and an $\mathrm{Fe}^{3+}$ ion on the $A$ site at $(1 / 81 / 81 / 8)$. Each of the conventional cells contains 56 atoms. Lattice and oxygen parameters were fixed at the values reported in the literature-i.e., $8.416 \AA$ and 0.380 , respectively. ${ }^{25}$ Calculations were performed for a $\mathrm{Zn}^{2+}$ ion on the $A$ site $\left(\mathrm{Zn}_{A}{ }^{2+}\right)$ and an $\mathrm{Fe}^{3+}$ ion on the $B$ site $\left(\mathrm{Fe}_{B}{ }^{3+}\right)$ in the normal spinel structure, and a $\mathrm{Zn}^{2+}$ ion on the $B$ site $\left(\mathrm{Zn}_{B}{ }^{2+}\right)$ and an $\mathrm{Fe}^{3+}$ ion on the $A$ site $\left(\mathrm{Fe}_{A}{ }^{3+}\right)$ in the disordered spinel structure. We also performed the calculation for a $\mathrm{Zn}_{A}{ }^{2+}$ in the disordered spinel structure in order to compare the theoretical spectrum with that for $\mathrm{Z}_{A}{ }^{2+}$ in the normal spinel structure. In each calculation, the initial ground state and the final transition state were computed separately. The final state is characterized by the excitation of an electron on a $1 s$ core orbital to unoccupied $4 p$ band at the $\mathrm{Zn}$ or Fe $K$ edge. Since a hole in the core orbital in the excited atom has some influence on the density of states, a more accurate computation is achieved by treating the presence of a core hole. Preliminary calculations revealed that when no core-hole effect is included, the calculated spectra are far from being satisfactory in reproducing the experimental XANES spectra, especially at the $\mathrm{Zn} K$ edge. Thus, we took fully into account the core-hole effect on the final state in the present calculation; an electron was removed from a core $1 s$ orbital to fill the lowest unoccupied $4 p$ orbital. The other conditions are as follows: the muffin-tin radii $R_{\mathrm{MT}}$ for $\mathrm{Fe}, \mathrm{Zn}$, and $\mathrm{O}$ were set to 1.75 a.u., and a product of muffintin radius and the maximum reciprocal space vector $K_{\max }$-i.e., the plane-wave cutoff- $R_{\mathrm{MT}} K_{\max }$, was fixed at 6.0 (atomic unit $\mathrm{Ry}^{1 / 2}$ ). The $k$-point sampling mesh in the reciprocal space was $3 \times 3 \times 3$. Theoretical XANES spectra were obtained by a product of the projected partial density of state ( $p$-PDOS) for unoccupied $4 p$ orbital in the final state and the radial part of the transition matrix elements. The $p$-PDOS was broadened by a Gaussian function of a full width at half maximum (FWHM) of $1 \mathrm{eV}$, as demonstrated in previous papers. ${ }^{21-24}$ The FWHM of $1 \mathrm{eV}$ was chosen for a fair comparison between experimental and calculated spectra; the use of the Gaussian function with larger FWHM, which tends to mask the fine details of theoretical spectra, may lead to an accidental agreement with experimental spectra. The theoretical transition energy was calculated as the difference in total energy between initial (ground) and final (core-hole) states.

We also analyzed the EXAFS spectra at the $\mathrm{Zn} K$ edge following well-established procedures. ${ }^{26}$ First, the extended absorption fine structure at the $\mathrm{Zn} K$ edge was isolated and normalized to the edge-step height and energy. The resultant data were then converted to photoelectron wave vector $(k)$ space and Fourier transformed to radial coordinates $(r$ space). The $k$ range of $3-12 \AA^{-1}$ with a $k^{3}$ weighting was employed in the Fourier transformation. At this stage in the analysis, the data were not corrected for electron phase shifts, and therefore, the Fourier peaks did not correspond directly to the bond distances of near neighbors but were instead shifted to lower $r$ values. The theoretical standards were generated by ab initio calculations using the FEFF code of Rehr et al. ${ }^{27}$ and fitted to the uncorrected experimental data in the $r$ space range of $1-4 \AA$.
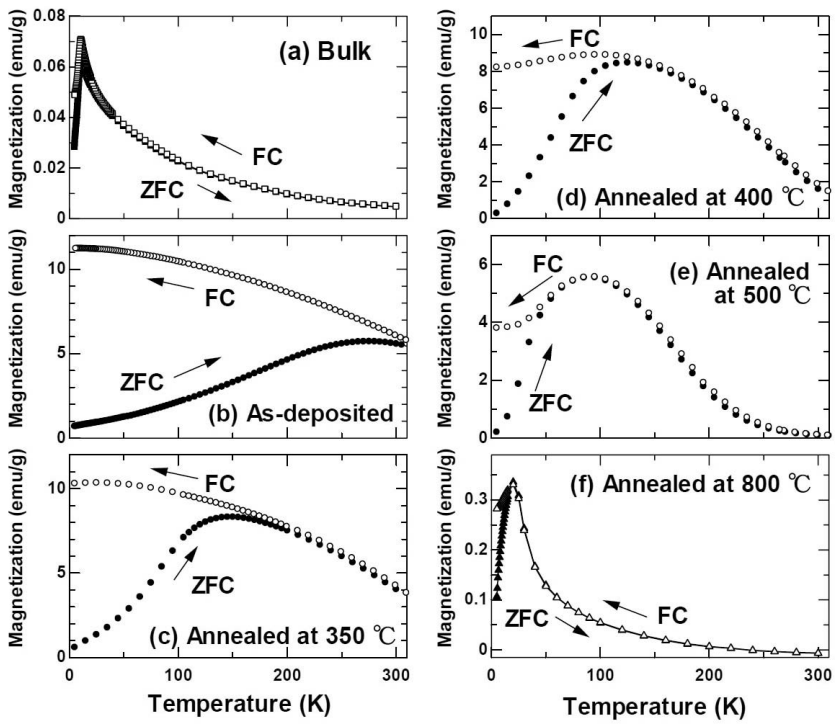

FIG. 2. Temperature dependences of ZFC (solid symbols) and FC (open symbols) magnetizations measured at an external magnetic field of $50 \mathrm{Oe}$ for (a) $\mathrm{ZnFe}_{2} \mathrm{O}_{4}$ bulk specimen, (b) asdeposited thin film, and thin films annealed at (c) $350{ }^{\circ} \mathrm{C}$, (d) $400{ }^{\circ} \mathrm{C}$, (e) $500{ }^{\circ} \mathrm{C}$, and (f) $800{ }^{\circ} \mathrm{C}$.

\section{RESULTS}

\section{A. Magnetic properties of $\mathrm{ZnFe}_{2} \mathrm{O}_{4}$}

Before discussing the main topic of this paper-i.e., XANES results-it is important to characterize our $\mathrm{ZnFe}_{2} \mathrm{O}_{4}$ samples, because the cation disorder of $\mathrm{ZnFe}_{2} \mathrm{O}_{4}$ is significantly affected by the synthetic procedures. Here, our $\mathrm{ZnFe}_{2} \mathrm{O}_{4}$ samples are characterized through the magnetic properties which are sensitive to the cation disorder. Figure 2 depicts the temperature variations of ZFC and FC magnetizations, $M_{\mathrm{ZFC}}(T)$ and $M_{\mathrm{FC}}(T)$, measured at an external magnetic field of 50 Oe for bulk specimen, as-deposited thin film, and the thin films annealed at temperatures between 350 and $800{ }^{\circ} \mathrm{C}$. The solid and open symbols in each of the figures represent the ZFC and FC magnetizations, respectively. For the bulk specimen as shown in Fig. 2(a), a cusp is observed at around $11 \mathrm{~K}$ in $M_{\mathrm{ZFC}}(T)$ and $M_{\mathrm{FC}}(T)$. Although the appearance of the cusp allows one to believe that $\mathrm{ZnFe}_{2} \mathrm{O}_{4}$ with normal spinel structure is antiferromagnetic with a Néel temperature of about $10 \mathrm{~K}$, recent studies of a single crystal using neutron scattering demonstrated that the long-range magnetic order is not attained in normal spinel $\mathrm{ZnFe}_{2} \mathrm{O}_{4}$ even at a very low temperature of $1.5 \mathrm{~K}$ or so because of three-dimensional geometrical spin frustration. ${ }^{1}$ Namely, normal spinel $\mathrm{ZnFe}_{2} \mathrm{O}_{4}$ crystal is not an antiferromagnet but an intrinsically spin-frustrated magnet. The presence of a small amount of structural disorder suppresses the spin frustration, leading to the finite-range magnetic order. This is signified by the discrepancy between $M_{\mathrm{ZFC}}(T)$ and $M_{\mathrm{FC}}(T){ }^{2}$ A close inspection at Fig. 2(a) reveals only a slight discrepancy between $M_{\mathrm{ZFC}}(T)$ and $M_{\mathrm{FC}}(T)$ at temperatures below the spin-freezing temperature. Thus, it is reasonable to assume that the cation distribution of bulk specimens is approximately equivalent to that of $\mathrm{ZnFe}_{2} \mathrm{O}_{4}$ with normal spinel structure. 
As for the as-deposited thin film [see Fig. 2(b)], on the other hand, a maximum in $M_{\mathrm{ZFC}}(T)$, which corresponds to the spin freezing, is observed at a temperature higher than room temperature, while $M_{\mathrm{FC}}(T)$ increases monotonically with decreasing temperature. The great discrepancy between $M_{\mathrm{ZFC}}(T)$ and $M_{\mathrm{FC}}(T)$ as well as the high spin-freezing temperature is mainly due to the formation of the metastable phase with a highly disordered cation distribution as will be mentioned below. Depending on the annealing temperature, the magnetic properties are gradually changed as shown in Figs. 2(c)-2(f). As the annealing temperature is increased, the spin-freezing temperature is shifted to lower temperatures accompanied by a decrease in magnetization in the measured temperature ranges. The $M_{\mathrm{ZFC}}(T)$ and $M_{\mathrm{FC}}(T)$ for the film annealed at $800{ }^{\circ} \mathrm{C}$ exhibit a behavior similar to that of bulk specimens, indicating that the annealing at $800{ }^{\circ} \mathrm{C}$ leads to an almost complete redistribution of $\mathrm{Zn}^{2+}$ and $\mathrm{Fe}^{3+}$ ions toward the normal spinel structure. Thus, it is considered that the variation of magnetic properties with annealing temperature reflects the gradual decrease in cation disorder, by which the superexchange interaction between $\mathrm{Fe}^{3+}$ ions in the $A$ and $B$ sites, $J_{A B}$, weakens to decrease the spin-freezing temperature and magnetization and instead the superexchange interaction between $\mathrm{Fe}^{3+}$ ions in the $B$ sites, $J_{B B}$, becomes dominant to bring about antiferromagnetic behavior.

\section{B. XANES spectra of $\mathrm{ZnFe}_{2} \mathrm{O}_{4}$}

For the aforementioned, well-characterized samples, we obtained experimental XANES spectra at $\mathrm{Zn}$ and $\mathrm{Fe} K$ edges. Figure 3 shows the experimental $\mathrm{Zn} K$-edge XANES spectra for the as-deposited thin film, the thin film annealed at $800{ }^{\circ} \mathrm{C}$, and the bulk specimen. The XANES spectra result from the parity-allowed $1 s-4 p$ transitions of $\mathrm{Zn}$. In Fig. 3 are also displayed the theoretical XANES spectra for $\mathrm{Zn}_{A}{ }^{2+}$ in ideal normal spinel $\mathrm{ZnFe}_{2} \mathrm{O}_{4}, \mathrm{Zn}_{A}{ }^{2+}$ in disordered $\mathrm{ZnFe}_{2} \mathrm{O}_{4}$, and $\mathrm{Zn}_{B}{ }^{2+}$ in disordered $\mathrm{ZnFe}_{2} \mathrm{O}_{4}$, which are represented by thin dashed-dotted, dashed, and solid lines, respectively. Theoretical transition energies are deviated from the experimental energy by $-37 \mathrm{eV}(\Delta E / E=0.38 \%)$, which are corrected in Fig. 3 by translation. The theoretical spectrum for $\mathrm{Zn}_{A}{ }^{2+}$ in ideal normal spinel $\mathrm{ZnFe}_{2} \mathrm{O}_{4}$ is essentially identical to that for $\mathrm{Zn}_{A}{ }^{2+}$ in the disordered $\mathrm{ZnFe}_{2} \mathrm{O}_{4}$ structure, indicating that the overall spectral shape is mainly determined by the first-nearest coordination structure. The experimental XANES spectra for $\mathrm{ZnFe}_{2} \mathrm{O}_{4}$ bulk specimen as depicted in the top of Fig. 3 can be decomposed into five peaks, denoted as $A-E$, and the relative positions and intensities of these peaks are well reproduced by the theoretical calculation for $\mathrm{Zn}_{A}{ }^{2+}$, indicating that $\mathrm{Zn}^{2+}$ ions occupy the $A$ sites in the bulk specimen. This is consistent with the result derived from the magnetic properties, as described in Sec. III A; namely, the cation distribution is approximately expressed as $\left[\mathrm{Zn}^{2+}\right]_{A}\left[\mathrm{Fe}_{2}{ }^{3+}\right]_{B} \mathrm{O}_{4}$-i.e., normal spinel $\mathrm{ZnFe}_{2} \mathrm{O}_{4}$. A close look at the top of Fig. 3 reveals that some additional peaks which are not observed in the experimental spectra appear in the theoretical spectra between peaks $C$ and $D$ as well as between peaks $D$ and $E$. An appropriate explanation for these

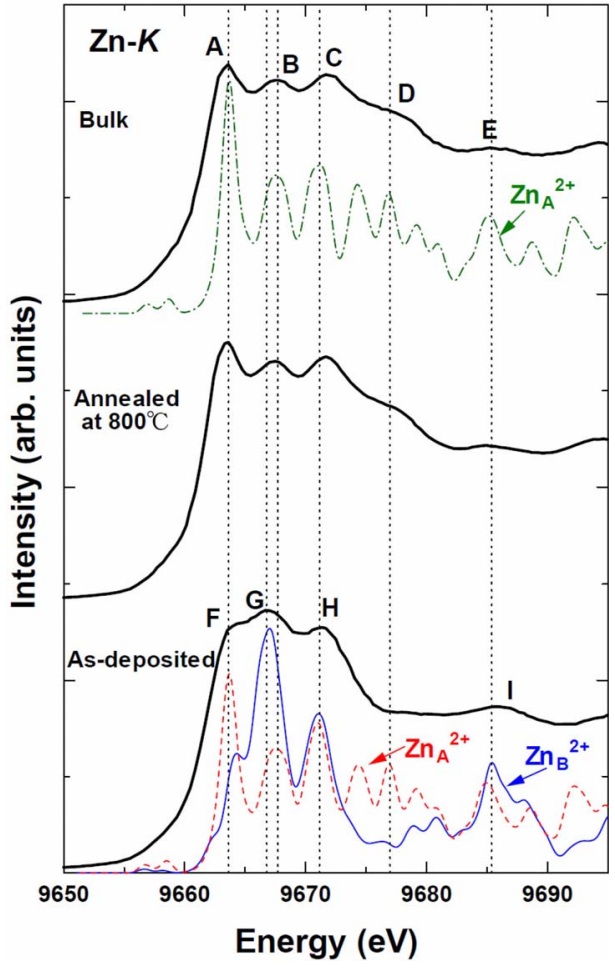

FIG. 3. (Color online) Comparison of experimental and theoretical $\mathrm{Zn}-K$ edge XANES spectra. From bottom to top, thick solid lines correspond to the experimental spectra for the as-deposited thin film, the thin film annealed at $800^{\circ} \mathrm{C}$, and the bulk specimen. Thin dashed-dotted, dashed, and solid lines represent theoretical spectra for $\mathrm{Zn}_{A}{ }^{2+}$ in the ideal normal spinel $\mathrm{ZnFe}_{2} \mathrm{O}_{4}, \mathrm{Zn}_{A}{ }^{2+}$ in the disordered $\mathrm{ZnFe}_{2} \mathrm{O}_{4}$, and $\mathrm{Zn}_{B}{ }^{2+}$ in the disordered $\mathrm{ZnFe}_{2} \mathrm{O}_{4}$, respectively. These theoretical spectra are shifted by an energy $\Delta E$ $=37 \mathrm{eV}(\Delta E / E=0.38 \%)$ in order to align the peak energies of the experimental spectra with those of the theoretical spectra.

peaks cannot be provided at present, but the use of larger supercell sizes may improve the theoretical spectrum. On the other hand, the experimental $\mathrm{Zn} K$-edge XANES spectra for the as-deposited $\mathrm{ZnFe}_{2} \mathrm{O}_{4}$ thin film, as shown in the bottom of Fig. 3, can be decomposed into four peaks. Apparent differences in the experimental spectra between as-deposited thin film and bulk specimen are as follows: (i) A peak at around $9667 \mathrm{eV}$ is the most intense in the as-deposited thin film as denoted by $G$, while the corresponding peak for the bulk specimen, denoted by $B$, is relatively weak. (ii) The transition energy of peak $G$ is slightly lower than that of peak $B$. (iii) A peak at around $9677 \mathrm{eV}$ is prominent only in the bulk specimen, as denoted by $D$. Thus, the experimental XANES spectra of as-deposited thin film cannot be reproduced by using only the theoretical spectrum for $\mathrm{Zn}_{A}{ }^{2+}$. The theoretical spectrum for $\mathrm{Zn}_{B}{ }^{2+}$ has an intense peak at around $9667 \mathrm{eV}$, which corresponds to peak $G$ in the experimental spectrum of as-deposited thin film. In addition, the theoretical spectrum for $\mathrm{Zn}_{B}{ }^{2+}$ manifests no detectable peak at around $9677 \mathrm{eV}$. Obviously, the calculation for $\mathrm{Zn}_{B}{ }^{2+}$ gives remarkable improvement in fitting the theoretical spectrum to the experimental one, although the agreement between the experimental data and calculation is not so good yet, especially at around the transition energy corresponding to peak 


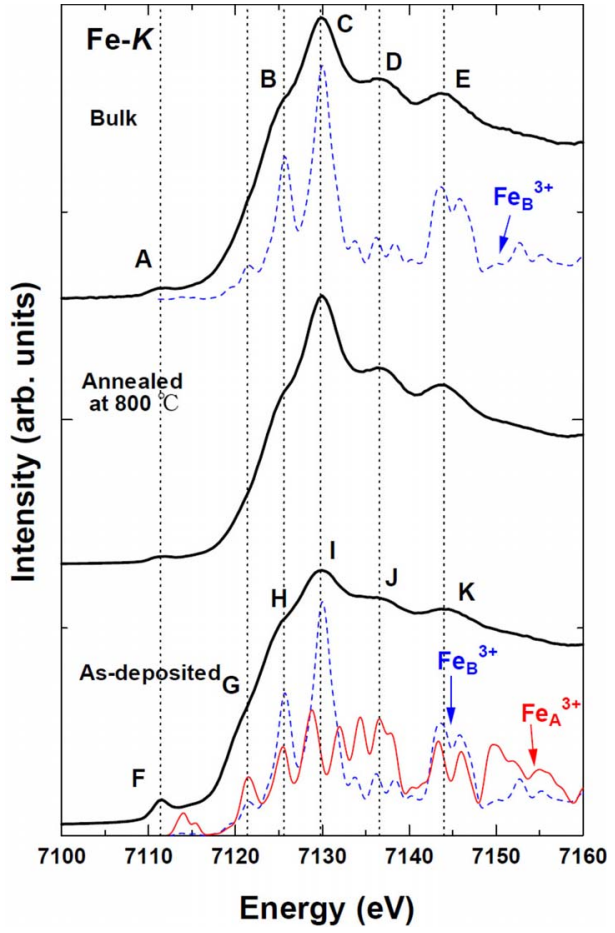

FIG. 4. (Color online) Comparison of experimental and theoretical Fe- $K$ edge XANES spectra. From bottom to top, thick solid lines correspond to the experimental spectra for the as-deposited thin film, the thin film annealed at $800{ }^{\circ} \mathrm{C}$, and the bulk specimen. Thin dashed and solid lines represent the theoretical spectra for $\mathrm{Fe}_{B}{ }^{3+}$ in ideal normal spinel $\mathrm{ZnFe}_{2} \mathrm{O}_{4}$ and $\mathrm{Fe}_{A}{ }^{3+}$ in the disordered $\mathrm{ZnFe}_{2} \mathrm{O}_{4}$, respectively. These theoretical spectra are shifted by an energy $\Delta E=24 \mathrm{eV}(\Delta E / E=0.34 \%)$ in order to align peak energies of the experimental spectra with those of the theoretical spectra.

$F$ in the experimental spectrum. The presence of peak $F$ can be explained if the calculated spectra for $\mathrm{Zn}_{A}{ }^{2+}$ and $\mathrm{Zn}_{B}{ }^{2+}$ are combined with a weighting as described below. As shown in the middle of Fig. 3, the experimental XANES spectra of the thin film annealed at $800{ }^{\circ} \mathrm{C}$ resembles that of the bulk specimen, and hence, the spectral features can be well reproduced by only the contribution from $\mathrm{Zn}_{A}{ }^{2+}$. This result again allows us to conclude that $\mathrm{ZnFe}_{2} \mathrm{O}_{4}$ with a random cation distribution is converted into that with normal spinel structure after annealing at $800{ }^{\circ} \mathrm{C}$.

Figure 4 presents the experimental $\mathrm{Fe} K$-edge XANES spectra, together with the theoretical spectrum calculated for $\mathrm{Fe}_{B}{ }^{3+}$ in the normal spinel structure (thin dashed line) and that for $\mathrm{Fe}_{A}^{3+}$ in the disordered spinel structure (thin solid line). The theoretical transition energy is shifted by $\Delta E$ $=24 \mathrm{eV}(\Delta E / E=0.34 \%)$ so that the most intense peak of the theoretical spectrum for $\mathrm{Fe}_{B}{ }^{3+}$ is aligned with that of experimental spectrum for bulk specimen. The Fe $K$-edge XANES spectrum of bulk specimen as depicted in the top of Fig. 4 can be decomposed into five peaks, denoted as $A-E$. When the theoretical spectrum is aligned with the experimental spectrum at the peak $C$, the energy of the peak $A$ is found to be higher than the experimental spectrum by $2 \mathrm{eV}$. Since the electronic states forming the peak $A$ are mainly Fe- $3 d$ states with a small portion of $\mathrm{Fe}-4 p$ states, an insufficient treatment of the electron correlation of $\mathrm{Fe}-3 d$ should be the major rea- son for the disagreement. ${ }^{21}$ Other than this point and the relative intensity of peak $D$, the overall spectral features of peaks $B-E$ are well reproduced by the theoretical spectrum for $\mathrm{Fe}_{B}{ }^{3+}$. This demonstrates that most of $\mathrm{Fe}^{3+}$ ions are located at $B$ sites in the spinel structure. Similarly to the case of $\mathrm{Zn} K$-edge XANES spectra, the Fe $K$-edge XANES spectral shape for the thin film annealed at $800{ }^{\circ} \mathrm{C}$ (see the middle of Fig. 4) is almost identical to that for the bulk specimen. For the as-deposited thin film as shown in the bottom of Fig. 4, on the other hand, the Fe $K$-edge XANES spectrum is decomposed into six peaks, denoted as $F-K$, and cannot be explained by considering only the contribution from $\mathrm{Fe}_{B}{ }^{3+}$. Spectral differences between the bulk specimen or the annealed thin film and as-deposited film are as follows. (i) The pre-edge peak at about $7112 \mathrm{eV}$ is relatively intense in the as-deposited thin film as denoted by $F$, compared to the corresponding peak for the bulk specimen, denoted by $A$. (ii) The shoulder peak at around $7120 \mathrm{eV}$, denoted by $G$, appears in the as-deposited thin film. (iii) The relative intensity of peaks at 7136 and $7144 \mathrm{eV}$ to that at $7130 \mathrm{eV}$ is more intense in the as-deposited thin film, as denoted by $J$ and $K$, than in the bulk specimen, as denoted by $D$ and $E$. The theoretical spectrum for $\mathrm{Fe}_{A}{ }^{3+}$ has a relatively intense pre-edge peak and exhibits some peaks at around 7120 and $7136 \mathrm{eV}$ which correspond to the peaks $G$ and $J$ for the as-deposited thin film, respectively. A combined sum of contributions from $\mathrm{Fe}_{A}{ }^{3+}$ and $\mathrm{Fe}_{B}{ }^{3+}$ is thus required to obtain a satisfactory agreement for the as-deposited thin film, indicating that some of the $\mathrm{Fe}^{3+}$ ions occupy the $A$ site in the spinel structure.

For the purpose of clarifying the degree of cation disordering in $\mathrm{ZnFe}_{2} \mathrm{O}_{4}$, we tried to combine the theoretical XANES spectra for $A$ - and $B$-site environments. Here, it should be stressed that when there exist crystallographic nonequivalent sites in a crystal, such as $\mathrm{Al}$ sites in garnet $\mathrm{Y}_{3} \mathrm{Al}_{5} \mathrm{O}_{12}$ crystal ${ }^{28}$ and $\mathrm{Si}$ sites in cubic spinel $\mathrm{Si}_{3} \mathrm{~N}_{4}$ crystal, ${ }^{29}$ a weighted sum of contributions from the different sites has been successful in reproducing the experimental XANES or ELNES spectra. Figure 5 shows the experimental $\mathrm{Zn} K$-edge XANES spectra for the as-deposited thin film and those annealed at elevated temperatures, along with the different weighted sums of contributions from theoretical spectra for $\mathrm{Zn}_{A}{ }^{2+}$ and $\mathrm{Zn}_{B}{ }^{2+}$ in the disordered $\mathrm{ZnFe}_{2} \mathrm{O}_{4}$. The combined spectrum with the cation distribution of $\left[\mathrm{Zn}_{0.4} \mathrm{Fe}_{0.6}\right]_{A}\left[\mathrm{Zn}_{0.6} \mathrm{Fe}_{1.4}\right]_{B} \mathrm{O}_{4}$ provides the best agreement with the measured spectrum of as-deposited thin film. The variation of XANES spectra with annealing temperature is also explained well by the gradual decrease in $x$ from 0.6 to 0 . As a result, $\mathrm{Zn} K$-edge XANES spectra can be utilized to determine $x$ in $\mathrm{ZnFe}_{2} \mathrm{O}_{4}$. In Fig. 6, Fe $K$-edge XANES spectra for the as-deposited thin film and those annealed at elevated temperatures are compared to the different weighted sums of contributions from theoretical spectra for $\mathrm{Fe}_{A}{ }^{3+}$ and $\mathrm{Fe}_{B}{ }^{3+}$ in disordered $\mathrm{ZnFe}_{2} \mathrm{O}_{4}$. Again, the relative intensities of peaks including pre-edge peaks for the experimental spectrum of as-deposited films are found to be reproduced by the theoretical spectrum at around $x=0.6$. Compared to the case of $\mathrm{Zn}$ $K$-edge XANES spectra, the variation of Fe $K$-edge XANES spectra with annealing temperature is small. This is presumably due to the fact that only $33 \%$ of $\mathrm{Fe}^{3+}$ ions occupy the $A$ 

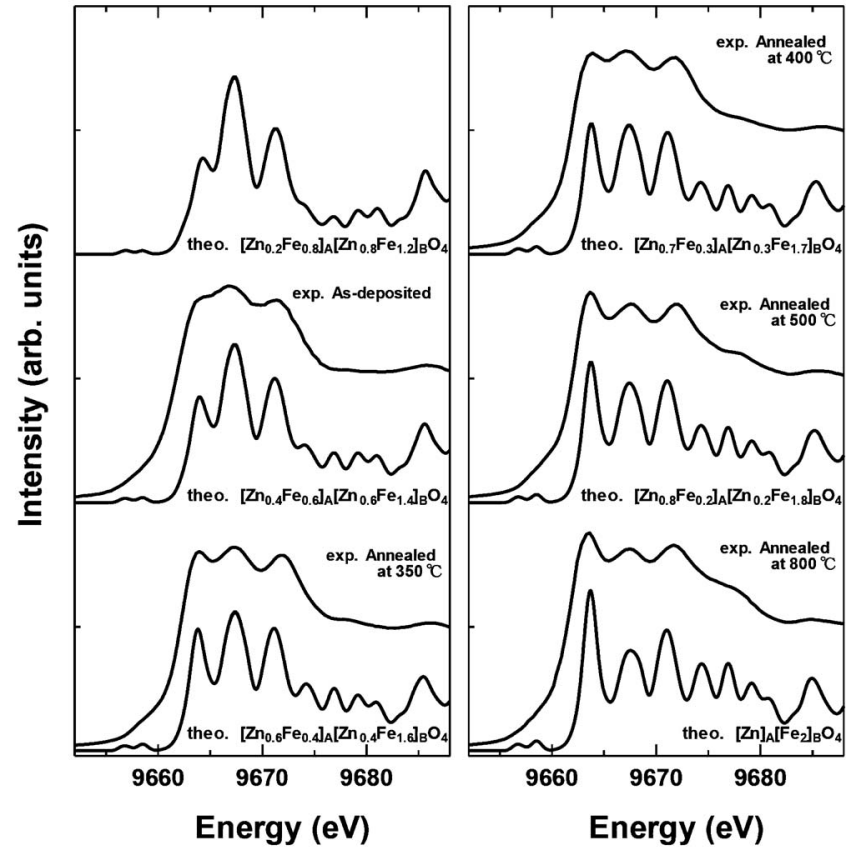

FIG. 5. Variation of experimental Zn $K$-edge XANES spectra with annealing temperature. Theoretical spectra obtained by different weighted sums of contributions from $\mathrm{Zn}^{2+}$ ions at $A$ and $B$ sites in the spinel structure are also shown for comparison.

sites in the completely disordered spinel structure. Namely, the contribution from $\mathrm{Fe}^{3+}$ ions in the $B$ sites dominates the Fe $K$-edge XANES spectra.

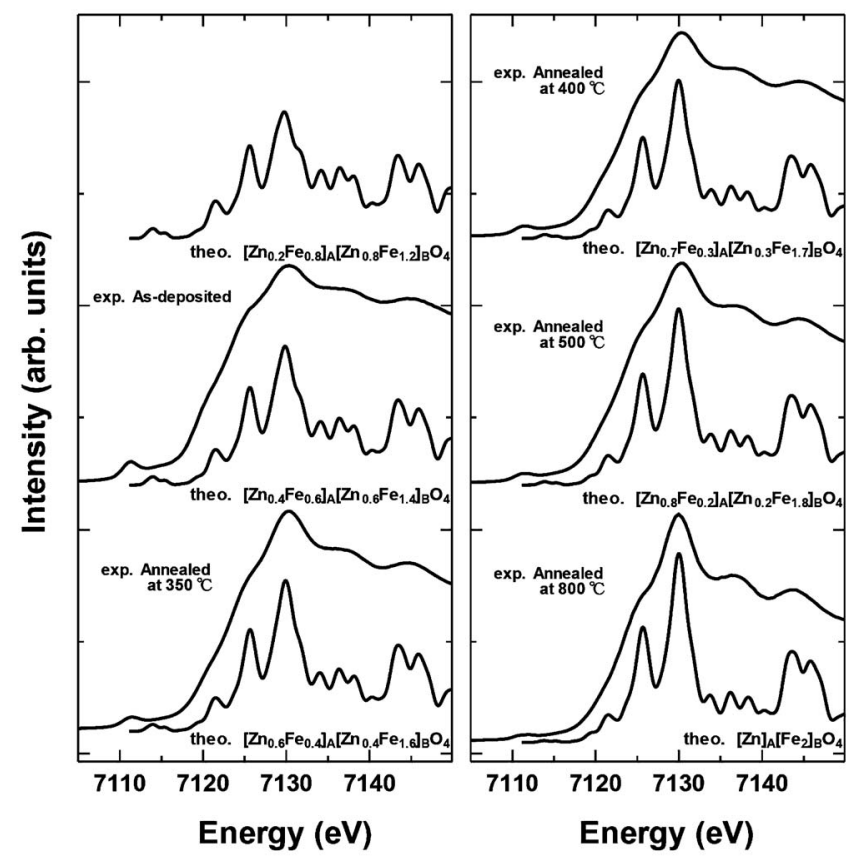

FIG. 6. Variation of experimental Fe $K$-edge XANES spectra with annealing temperature. Theoretical spectra obtained by different weighted sums of contributions from $\mathrm{Fe}^{3+}$ ions at the $A$ and $B$ sites in the spinel structure are also shown for comparison.

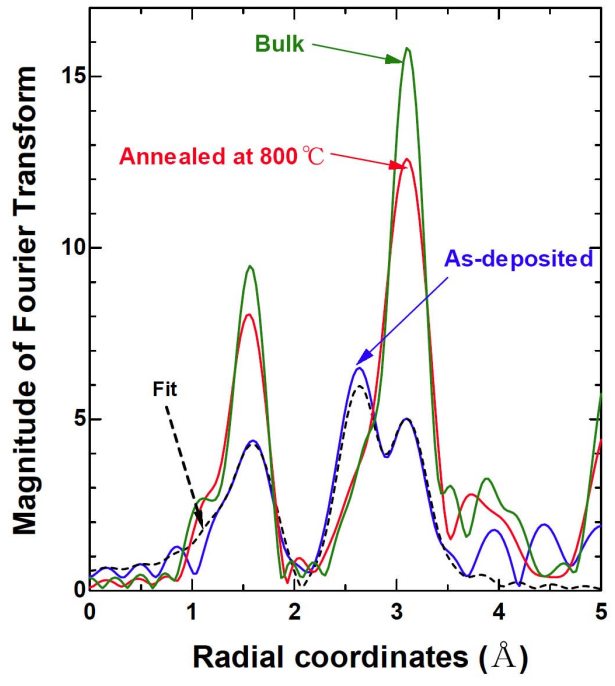

FIG. 7. (Color online) Fourier transforms of Zn- $K$ EXAFS data collected from the bulk specimen, the as-deposited thin film, and the thin film annealed at $800{ }^{\circ} \mathrm{C}$ (solid lines). Corrections for phase shift have not been included in the Fourier transformation. The dashed line is a theoretical one showing the best fit to the experimental data in the range of $1-4 \AA$ for the Fourier transform of as-deposited thin film.

\section{EXAFS analysis}

In this section, we perform an EXAFS analysis in order to manifest the validity of $x$ values derived from XANES calculations. As previously reported for $\mathrm{ZnFe}_{2} \mathrm{O}_{4}$, the EXAFS analysis of $\mathrm{Zn} K$-edge spectra was found to be even more straightforward compared to that of $\mathrm{Fe} K$-edge spectra. ${ }^{4,17}$ Figure 7 displays the Fourier transforms of $\mathrm{Zn} K$-edge EXAFS spectra for the as-deposited thin film, thin film annealed at $800{ }^{\circ} \mathrm{C}$, and the bulk specimen. Considerable differences between the bulk specimen or the annealed thin film and as-deposited thin film are found in the peaks due to secondnearest neighbors in the range of 2-4 $\AA$. The shape of the peaks in this range depends on whether $\mathrm{Zn}^{2+}$ ions occupy $A$ or $B$ sites in the spinel structure. The Fourier transforms for bulk specimen and annealed thin film show just one intense peak at around 3.1 $\AA$. Similar results have been observed for polycrystalline $\mathrm{ZnFe}_{2} \mathrm{O}_{4}$ produced by the solid-state reaction. ${ }^{6,8}$ The peak observed at $3.1 \AA$ originates from $\mathrm{Zn}_{A}{ }^{2+}-\mathrm{Zn}_{A}{ }^{2+}, \mathrm{Zn}_{A}{ }^{2+}-\mathrm{Fe}_{B}{ }^{3+}$, and $\mathrm{Zn}_{A}{ }^{2+}-\mathrm{O}^{2-}$ pairs, which is indicative of the normal spinel structure where $\mathrm{Zn}^{2+}$ ions reside on the $A$ sites. For as-deposited thin film, on the other hand, two peaks were observed at around 2.6 and $3.1 \AA$. The peak observed at $2.6 \AA$ corresponds to the contributions from $\mathrm{Zn}_{B}{ }^{2+}-\mathrm{Zn}_{B}{ }^{2+}$ and $\mathrm{Zn}_{B}{ }^{2+}-\mathrm{Fe}_{B}{ }^{3+}$ pairs and gives evidence for the occupancy of $B$ sites by $\mathrm{Zn}^{2+}$ ions, as demonstrated in EXAFS analyses on ultrafine $\mathrm{ZnFe}_{2} \mathrm{O}_{4}$ particles prepared by the coprecipitation method ${ }^{6}$ and mechanical-milled $\mathrm{ZnFe}_{2} \mathrm{O}_{4}$ powder ${ }^{4}$ as well as on spinel ferrites such as spin-sprayed NiZn-ferrite ${ }^{14}$ and MnZn-ferrite nanoparticles prepared by the reverse micellar method. ${ }^{16} \mathrm{~A}$ feature characteristic of the present as-deposited $\mathrm{ZnFe}_{2} \mathrm{O}_{4}$ thin film is that the relative amplitude of the peak at around $2.6 \AA$ to that at around $3.1 \AA$ is much stronger compared to the results for the $\mathrm{ZnFe}_{2} \mathrm{O}_{4}$ 
specimens as reported previously. ${ }^{4,6,8}$ This can be related to such a spinel-type structure that a rather large fraction of $\mathrm{Zn}^{2+}$ ions is present in the $B$ sites. Following the method as described in Ref. 14, a least-squares fitting was performed over the uncorrected $r$-space range of 1.0-4.0 $\AA$ for the Fourier transform of the as-deposited thin film. The theoretical standards for $\mathrm{Zn}_{A}{ }^{2+}$ and $\mathrm{Zn}_{B}{ }^{2+}$ in the spinel $\mathrm{ZnFe}_{2} \mathrm{O}_{4}$ were generated by $a b$ initio calculations using the FEFF code of Rehr et $a .^{27}$ Here, $\mathrm{Zn} K$-edge EXAFS analysis is refined using the ATHENA and ARTEMIS code of Ravel ${ }^{30}$ and Newville, ${ }^{31}$ respectively, in order to estimate the cation distribution of as-deposited $\mathrm{ZnFe}_{2} \mathrm{O}_{4}$ thin film. The best fit to the experimental data is shown by a dashed curve in the bottom of Fig. 7. Approximately $64 \%$ of $\mathrm{Zn}^{2+}$ ions are found to occupy the $B$ sites in the spinel-type structure. Namely, the cation distribution of the as-deposited thin film is represented as $\left[\mathrm{Zn}_{0.36}{ }^{2+} \mathrm{Fe}_{0.64}{ }^{3+}\right]_{A}\left[\mathrm{Zn}_{0.64}{ }^{2+} \mathrm{Fe}_{1.36}{ }^{3+}\right]_{B} \mathrm{O}_{4}$, which is close to the spinel structure with a completely random cation distribution $(x=2 / 3)$. Consequently, the $x$ values derived from XANES simulations agree well with those from the EXAFS analysis, indicating that the XANES calculations are useful for determining the degree of cation disordering in the spinel structure.

\section{DISCUSSION AND CONCLUSIONS}

From the theoretical simulations of XANES and EXAFS spectra at the $\mathrm{Zn} K$ edge, it is concluded that about $60 \%$ of $\mathrm{Zn}^{2+}$ ions reside on the $B$ sites in the as-deposited $\mathrm{ZnFe}_{2} \mathrm{O}_{4}$ thin film, despite a strong tendency for $\mathrm{Zn}^{2+}$ ions to occupy the $A$ sites in the spinel-type structure. The occupancy of $B$ sites by $\mathrm{Zn}^{2+}$ ions displaces some $\mathrm{Fe}^{3+}$ ions from the $B$ to $A$ sites to produce the disordered distribution of $\mathrm{Fe}^{3+}$ ions as demonstrated in the spectral analysis of Fe $K$-edge XANES spectra (see Figs. 4 and 6). The magnetic properties of $\mathrm{ZnFe}_{2} \mathrm{O}_{4}$ are significantly affected by the cation disordering, depending on $x$, as shown in Fig. 2. If $x$ is very small, the magnetic structure is still dominated by the superexchange interaction between $\mathrm{Fe}^{3+}$ ions on the $B$ sites, $J_{B B}$, and antiferromagnetic behavior is observed. As $x$ is increased over 0.33 , the superexchange interaction between $\mathrm{Fe}^{3+}$ ions in $A$ and $B$ sites, $J_{A B}$, becomes dominant, ${ }^{32}$ and hence, ferrimagnetic behavior appears. A further increase in $x$ strengthens $J_{A B}$ to bring about a large magnetization even at room temperature. ${ }^{9}$ Such a highly disordered cation distribution, however, gives rise to a frustration due to the competition between $J_{A B}$ and $J_{B B}$. As a result, the as-deposited thin film exhibits the magnetic transition as observed for cluster spinglass systems, as can be seen from Fig. 2(b) - that is, a discrepancy between $M_{\mathrm{ZFC}}(T)$ and $M_{\mathrm{FC}}(T)$. The spin-freezing phenomenon above room temperature is caused by the strong $J_{A B}$, because the spin-freezing temperature is considered to be proportional to the strength of the superexchange interaction. Our previous results of linear and nonlinear ac susceptibility for as-deposited thin film also showed that superparamagnetic blocking of the clusters accompanied by intercluster interactions takes place at around the magnetic transition temperature $(\sim 325 \mathrm{~K}) .{ }^{9}$ The rather high degree of disorder may be characteristic of the thin-film structure that results from nonequilibrium processing such as a sputtering method and a PLD technique. In fact, it has been reported for some spinel-type oxides that the site distribution in thin-film structure prepared via vapor phases is largely different from that in the stable phase. ${ }^{33}$ XANES analyses utilizing firstprinciples calculations that include the core-hole effect, when combined with other techniques, promise to be an important tool in determining the structure of magnetic materials.

\section{ACKNOWLEDGMENTS}

This work was financially supported by a Grant-in-Aid for Scientific Research (No. 18360316) from the Ministry of Education, Culture, Sports, Science, and Technology (MEXT), Japan, and by the Yazaki Foundation. One of authors (S.N.) acknowledges a Grant-in-Aid (No. 1701800) from the Japan Society for the Promotion of Science (JSPS).
*Corresponding author: fujita@dipole7.kuic.kyoto-u.ac.jp

${ }^{1}$ K. Kamazawa, Y. Tsunoda, H. Kadowaki, and K. Kohn, Phys. Rev. B 68, 024412 (2003).

${ }^{2}$ T. Usa, K. Kamazawa, H. Sekiya, S. Nakamura, Y. Tsunoda, K. Kohn, and M. Tanaka, J. Phys. Soc. Jpn. 73, 2834 (2004).

${ }^{3}$ C. N. Chinnasamy, A. Narayanasamy, N. Ponpandian, K. Chattopadhyay, H. Guerault, and J.-M. Greneche, J. Phys.: Condens. Matter 12, 7795 (2000).

${ }^{4}$ S. A. Oliver, V. G. Harris, H. H. Hamdeh, and J. C. Ho, Appl. Phys. Lett. 76, 2761 (2000).

${ }^{5}$ T. Sato, K. Haneda, M. Seki, and T. Iijima, Appl. Phys. A: Solids Surf. 50, 13 (1990).

${ }^{6}$ B. Jeyadevan, K. Tohji, and K. Nakatsuka, J. Appl. Phys. 76, 6325 (1994).

${ }^{7}$ K. Tanaka, Y. Nakahara, K. Hirao, and N. Soga, J. Magn. Magn. Mater. 131, 120 (1994).

${ }^{8}$ K. Tanaka, M. Makita, Y. Shimizugawa, K. Hirao, and N. Soga, J.
Phys. Chem. Solids 59, 1611 (1998).

${ }^{9}$ S. Nakashima, K. Fujita, K. Tanaka, and K. Hirao, J. Phys.: Condens. Matter 17, 137 (2005).

${ }^{10}$ K. Tanaka, S. Nakashima, K. Fujita, and K. Hirao, J. Phys.: Condens. Matter 15, L469 (2003).

${ }^{11}$ S. Nakashima, K. Fujita, K. Tanaka, and K. Hirao, J. Ceram. Soc. Jpn. 112, S961 (2004).

${ }^{12}$ K. Tanaka, S. Nakashima, K. Fujita, and K. Hirao, J. Appl. Phys. 99, 106103 (2006).

${ }^{13}$ Y. Yamamoto, H. Tanaka, and T. Kawai, Jpn. J. Appl. Phys., Part 2 40, L545 (2001).

${ }^{14}$ V. G. Harris, N. C. Koon, C. M. Williams, Q. Zhang, M. Abe, and J. P. Kirkland, Appl. Phys. Lett. 68, 2082 (1996).

${ }^{15}$ S. A. Morrison, C. L. Cahill, E. E. Carpenter, S. Calvin, R. Swaminathan, M. E. McHenry, and V. G. Harris, J. Appl. Phys. 95, 6392 (2004).

${ }^{16}$ S. Calvin, E. E. Carpenter, V. G. Harris, and S. A. Morrison, 
Appl. Phys. Lett. 81, 3828 (2002).

${ }^{17}$ S. Calvin, E. E. Carpenter, B. Ravel, V. G. Harris, and S. A. Morrison, Phys. Rev. B 66, 224405 (2002).

${ }^{18}$ A. Yang, Z. Chen, X. Zuo, D. Arena, J. Kirkland, C. Vittoria, and V. G. Harris, Appl. Phys. Lett. 86, 252510 (2005).

${ }^{19}$ S. D. Mo and W. Y. Ching, Phys. Rev. B 62, 7901 (2000).

${ }^{20}$ S. D. Mo and W. Y. Ching, Appl. Phys. Lett. 78, 3809 (2001).

${ }^{21}$ T. Yamamoto, T. Mizoguchi, and I. Tanaka, Phys. Rev. B 71, 245113 (2005).

${ }^{22}$ T. Mizoguchi, I. Tanaka, S. Yoshioka, M. Kunisu, T. Yamamoto, and W. Y. Ching, Phys. Rev. B 70, 045103 (2004).

${ }^{23}$ M. Kunisu, F. Oba, H. Ikeno, I. Tanaka, and T. Yamamoto, Appl. Phys. Lett. 86, 121902 (2005).

${ }^{24}$ I. Tanaka, T. Mizoguchi, M. Matsui, S. Yoshioka, H. Adachi, T. Yamamoto, T. Okajima, M. Umesaki, W. Y. Ching, Y. Inoue, M. Mizuno, H. Araki, and Y. Shirai, Nat. Mater. 2, 541 (2003).

${ }^{25}$ O. Hajicek, Hutnické Listy 3, 265 (1948).
${ }^{26}$ D. E. Sayers and B. A. Bunker, X-ray Absorption: Principles, Applications. Techniques of EXAFS, SEXAFS and XANES (Wiley, New York, 1988), Vol. 92, pp. 211-253.

${ }^{27}$ J. J. Rehr, R. C. Albers, and S. I. Zabinsky, Phys. Rev. Lett. 69, 3397 (1992).

${ }^{28}$ Y.-N. Xu, Y. Chen, S.-D. Mo, and W. Y. Ching, Phys. Rev. B 65, 235105 (2002).

${ }^{29}$ I. Tanaka, T. Mizoguchi, T. Sekine, H. He, K. Kimoto, T. Kobayashi, S. D. Mo, and W. Y. Ching, Appl. Phys. Lett. 78, 2134 (2001).

${ }^{30}$ B. Ravel and M. Newville, Phys. Scr. T115, 1007 (2005).

${ }^{31}$ M. Newville, J. Synchrotron Radiat. 8, 322 (2001).

${ }^{32}$ J. Hubsch, G. Gavoille, and J. Bolfa, J. Appl. Phys. 49, 1363 (1978).

${ }^{33}$ X. Zuo, A. Yang, J. A. Christodoulides, V. G. Harris, and C. Vittoria, Appl. Phys. Lett. 87, 152505 (2005). 